\section{Improving Travel-Time Reliability by the Use of Trip Booking}

\author{
Ronald de Feijter, Joseph J. M. Evers, and Gabriel Lodewijks
}

\begin{abstract}
The congestion of our infrastructure, particularly (urban) motorways, continues to increase. Efficient planning, for instance in freight transport, is hindered by the resulting unreliability of travel times. Another effect of this congestion is a reduced utilization rate of the road. This paper presents trip booking, a method aimed at improvement of the reliability of travel times as well as an increase in the effective use of road capacity. Increased reliability facilitates better logistic planning. Furthermore, it allows the sharing of infrastructure between different modalities, with each modality having its own operational time window. The system aims at open dedicated infrastructure, such as bus lanes and dedicated freight lanes, and preserves the autonomy of both the provider and user of the infrastructure. The advantage claims are supported by simulation results for basic network configurations.
\end{abstract}

Index Terms-Automated highways, communication, simulation of traffic flow, traffic control, transportation.

\section{INTRODUCTION}

During the last decades, (urban) motorways have become considerably crowded. Moreover, the need for transport is expected to continue to increase in coming decades. Major contributors to this effect are population growth and our more and more transport-dependent lifestyles [1]. As a result of heavy road use, congestion occurs. Research among trucking company managers [2] states that the most problematic aspect of congestion is unreliable travel times. Travel-time reliability also is a major issue in the situation that occurs when different modalities share infrastructure capacity. A proposal for alternative use of the "Betuwelijn" railroad in The Netherlands [3] suggests that, in addition to traditional freight trains, hybrid vehicles (vehicles that are able to travel on road and rail) should operate on the railroad. In this context, hybrid vehicles should not interfere with the operation of freight trains. Therefore, each vehicle that enters the railroad must be sure to leave that railroad before some future point in time. This condition requires a reliable travel time.

Research among trucking company managers as well as the proposal regarding dual use of infrastructure shows the need for a method to improve travel-time reliability. To that end, this paper introduces a system called trip booking. Trip booking is a reservation mechanism for the use of infrastructure capacity. The system is intended for open dedicated infrastructure. "Dedicated" indicates that only a certain class of vehicles is allowed to use this infrastructure and "open" indicates that anyone is allowed to use the infrastructure as long as their vehicle satisfies the conditions specifying the class to which the infrastructure is dedicated. These conditions may indicate minimum speed, acceleration, deceleration, and communication equipment requirements. Examples of such infrastructure are bus lanes, dedicated freight lanes, and the infrastructure proposed in the DistriRoad concept [4]. The open dedicated admittance strategy could also be applied to underground freight transportation systems [5]. An essential property of trip booking is the high

Manuscript received November 26, 2003; revised July 15, 2004 and August 10, 2004. The Associate Editor for this paper was F.-Y. Wang.

R. de Feijter and G. Lodewijks are with Faculty Design, Engineering and Production, Section Transport Engineering and Logistics, Delft University of Technology, Delft 2628 CD, The Netherlands (e-mail: R.deFeijter@wbmt.tudelft.nl).

J. J. M. Evers is with Evers R\&D Logistic, Oegstgeest 2343JH, The Netherlands.

Digital Object Identifier 10.1109/TITS.2004.837817 degree of autonomy that it preserves for both the user and supplier of the infrastructure, while at the same time providing reliable information about trip duration and future capacity use.

The objective of this paper is to present the trip-booking system, which improves the reliability and predictability of travel times. Besides travel-time reliability, trip booking is also shown to result in an increase in the effective use of road capacity. The concept of trip booking, the required communications (what will be communicated and when), and underlying algorithms (what to do with the received information) are described. The improvement claims are advocated by simulation experiments. Performance is, among others, measured in average waiting times and flow rates. Improving travel-time reliability and effective use of road capacity while preserving autonomy of the actors constitutes the main contribution of this paper. Hardware implementations for the vehicle and infrastructure are not discussed.

This paper is organized as follows. Section II refers to previous work done in other studies. Section III elaborates on the operation of trip booking, communication schemes, and algorithms. The simulation model, simulation experiments, and their results are discussed in Sections IV and V, after which Section VI puts trip booking in a wider perspective. Finally, the conclusion follows in Section VII.

\section{HIGHWAY-CAPACITY BOOKING IN THE LITERATURE}

The concept behind trip booking (advance reservation of a place on the highway) has been mentioned before in the literature, but neither extensively nor neither recently. Akahane and Kuware [6] used a stated-preference (SP) survey to evaluate the user acceptance and effectiveness of a trip-reservation system aimed at relieving holiday traffic congestion. They concluded that the system seemed promising. Wong [7] provides an overview of the advantages, components, and difficulties of a system for advance booking for highway use. He concludes that the system he proposes is still in its infancy, but has great potential. Koolstra [8] stresses the advantages of slot-reservation systems over congestion pricing and supports this claim with a comparative analysis. While these papers mainly indicate the usefulness of such a reservation system and which elements should be part of it, this paper goes into detail on how such a system could actually be implemented (in terms of required communications and algorithms). The applicability of the proposed implementation is shown with simulation experiments, which further support the advantages of such a reservation system. This paper focuses instead on using the reservation system for trucks and buses, whereas the mentioned references focus on passenger cars. The resulting system is, however, not limited to application to trucks and buses only.

Although a highway-reservation system can be used as an independent application, it can also be used in cooperation with other technologies. Ramp-metering systems, which are used to control the vehicle flow at highway on ramps, have been studied extensively, for instance in [9] and [10]. Various studies, such as [11], indicate that the performance of coordinated ramp-metering algorithms can be improved by improving the quality of the origin-destination (OD) estimation. A highway-reservation system can provide a very reliable OD estimation (since the users have indicated their origin and destination for their reservation) and can, therefore, very well be used in cooperation with up-to-date ramp-metering algorithms. In [12], destination-specific metering at on ramps is discussed as a strategy for freeway congestion mitigation and, although not mentioned, a reservation system could support such destination-specific metering by providing the intended vehicle destinations. A reservation system as presented in this paper also fits within the current developments on automated highway systems (AHS) [13]. 
TABLE I

TRIP-BOOKING PROCEDURE

\begin{tabular}{|c|c|}
\hline Moment & Action \\
\hline $\begin{array}{l}\text { Prior to } \\
\text { intended } \\
\text { departure } \\
\text { (e.g. some } \\
\text { hours) }\end{array}$ & $\begin{array}{l}\text { The transport provider sends a trip request to the } \\
\text { infrastructure manager. The infrastructure manager } \\
\text { allocates a slot (a specific departure time) to this } \\
\text { request and returns a ticket within one minute of the } \\
\text { request. The transport provider either accepts or } \\
\text { rejects the ticket. (Cancellation of an accepted ticket } \\
\text { at a later time is possible, but at a cost.) }\end{array}$ \\
\hline $\begin{array}{l}\text { When the } \\
\text { vehicle shows } \\
\text { up for } \\
\text { departure }\end{array}$ & $\begin{array}{l}\text { The vehicle reports its presence to the infrastructure } \\
\text { manager. The infrastructure manager allocates an } \\
\text { actual departure time to the vehicle. At this allocated } \\
\text { time the vehicle enters the network. This allocation } \\
\text { takes into account the current situation on the } \\
\text { infrastructure and the pending reservations. }\end{array}$ \\
\hline $\begin{array}{l}\text { During the } \\
\text { trip }\end{array}$ & $\begin{array}{l}\text { The vehicle notifies the network manager when a } \\
\text { link is entered or left. The infrastructure manager } \\
\text { uses this information in the actual departure time } \\
\text { allocations. }\end{array}$ \\
\hline
\end{tabular}

\section{TRIP-BOOKING CONCEPT}

In the field of logistics, reservation is an often-applied method to guarantee future availability of capacity. This is a major aid in reliable planning. Examples can, for instance, be found in air traffic (dynamic departure slot reservation) [14] and telecommunications (bandwidth reservations). Reservation systems are also scattered through everyday life, such as in restaurants (table reservation) and airplanes (seat reservation). Trip booking applies the reservation concept to infrastructure capacity such as roads. This section elaborates on the concept of trip booking.

Trip booking is applied to transport networks. Such a network consists of nodes, links, and routes. Nodes at which the network can be entered or left are referred to as sites. Links are located between two nodes and facilitate one-way travel from the origin node to the destination node. A route is a sequence of links. For simplicity, only one route is assumed to exist between two sites in each direction. Trips are performed nonstop, i.e., the vehicles traverse the links on the route without stopping at any node. The capacity of a link is defined by the number of vehicles that are able to enter the link within a period of time. The capacity of a route is determined by the capacity of the links on that route. This capacity depends on the maximum speed, size of the vehicles, normal safe following distance, and the algorithm used for distance control. Two distinct actors can be distinguished in trip booking: the supplier of the infrastructure capacity (the infrastructure manager) and the user of the infrastructure (the transport provider, whose vehicles actually use the infrastructure).

The trip-booking procedure is summarized in Table I. The trip request includes the desired departure time, route, shift directions, and possible additional information (e.g., regarding priority). The request is sent by the transport provider to the infrastructure manager (e.g., by Internet). The shift directions indicate how the departure time may be shifted if a slot at the desired departure time is not available. These shift directions may indicate earlier, later, as close as possible to the desired departure time or more complicated directions. The infrastructure manager uses a slot-allocation algorithm, which respects the shift directions, to allocate a departure time to the vehicle. Fig. 1 shows a simple first-come first-served algorithm to perform this task. For this allocation, the capacity of each link is divided into discrete time periods, referred to as capacity periods. For instance, the capacity of a link that allows access to 0.4 vehicles/s can be divided into capacity periods of 1 min with a capacity of 24 vehicles each (assuming that all vehicles are

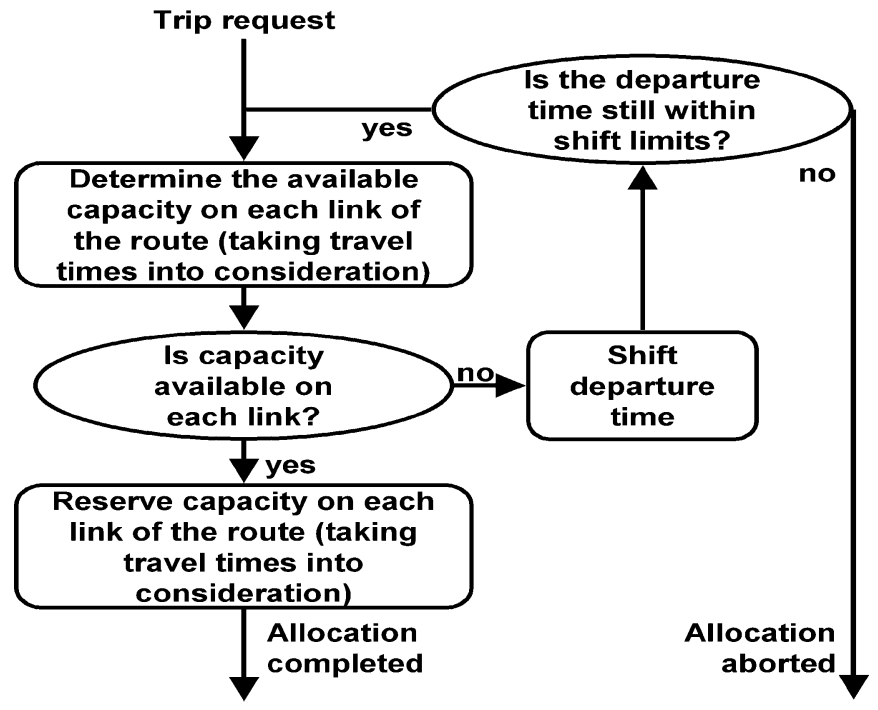

Fig. 1. Simple first-come first-served allocation algorithm used by the infrastructure manager to allocate slots to trip requests.

of the same type). The capacity period, to which a vehicle's desired departure time belongs, is determined at the start of the reservation procedure. This concerns a capacity period on the starting link. Subsequently, the corresponding capacity periods for all following links can be determined and whether there is capacity left during these periods. Finally, if the capacity along the entire route is available, the capacity will be reserved and a slot (i.e., a specific departure time) within the capacity period on the starting link is attached to a ticket, which is sent to the vehicle (or its representative). More elaborate algorithms may be used, which could include priority rules such as the bucket-control mechanism [15]. Once the vehicle shows up for departure, an actual departure time is assigned to it by an allocation algorithm, which can be virtually similar to the original reservation algorithm. This second allocation, referred to as trip metering, uses the current and future network situation (based on the reservations) as input and provides a mechanism to respond to up-to-date information. Only vehicles with a valid ticket receive an actual departure time. Vehicles without a valid ticket receive a ticket for the first available time before allocation of an actual departure time. The ticket of a vehicle is valid if the vehicle shows up within a certain time limit of the allocated slot time. Reliability of the reservations can be supported by a pricing system that supports the desired use of the reservation system.

The most obvious effect of trip booking is the relocation of waiting times when the traffic volume exceeds the road capacity. In the (unorganized) reference situation, this would result in increased travel times, whereas with trip booking this would result in a shifting departure time. With ramp metering, the waiting times would occur at the road entrance or network entrance. In the reference situation, they would occur "on the road." However, since the waiting time in the trip-booking situation is known as soon as the reservation is made, this time could be usefully spend. In this context, it is important to note that the average anticipation time, which refers to the time between the issuance of the request and the desired departure time, is much larger than the average shift time. The planning can be made (or adjusted) to fit the allocated departure time. It is also possible that the departure time will shift to an earlier time. Therefore, this time might no longer be considered as waiting time. A less obvious effect is that the traffic density can be kept at a rate that allows maximum throughput according to the Fundamental Traffic Characteristic [16]. As a result of trip booking, the travel times will be smaller than (or at maximum equal to) the travel times in the reference situation. Furthermore, travel times will be more reliable, since 
TABLE II

PERFORMANCE INDICATORS

\begin{tabular}{ll}
\hline \hline \multicolumn{1}{c}{ Indicator } & \multicolumn{1}{c}{ Description } \\
\hline $\begin{array}{l}\text { Average } \\
\text { waiting time }\end{array}$ & Average waiting time \\
$\begin{array}{l}\text { Logistic } \\
\text { waiting time }\end{array}$ & $5 / 6^{\text {th }}$ quantile of the waiting time \\
Flow rate & $\begin{array}{l}\text { Mean realized flow divided by the maximum } \\
\text { possible flow } \mathrm{x} 100 \%\end{array}$ \\
\hline \hline
\end{tabular}

congestion will not occur. Using only the allocation algorithm at the entrance without prior reservation (trip metering without reservation) is similar to ramp metering with OD information. Trip metering also shows similarities with the traffic-flow management problem (TFMP), which involves departure-time adjustments of planes to avoid congestion in the air [17]. This system of allowing entrance only to vehicles that have a "free path" prevents blocking: a queue resulting from congestion on a part of a route may block vehicles that only use another part of that route. This blocking restricts the throughput of the routes covered by these vehicles, resulting in an ineffective use of road capacity. Trip metering (and, thus, trip booking) therefore increases the effective use of road capacity. This effect will be illustrated in the next section.

In Section I, the situation has been mentioned in which the infrastructure has to be shared with other modalities, such as trains. This can be easily arranged by reserving the time slots required for these trains prior to releasing the capacity to normal vehicle reservations. Transfer capacity can also be included without difficulty. Transfer links can just be regarded as transport links with transfer time instead of travel time. They can then be included like any other link. If the network includes several possible routes between two nodes, the allocation algorithm could take care of the route choice (depending on the available capacity) and the request should rather indicate the origin and destination than the route. Another extension of the allocation algorithm would be to support the use of platoons. The current description allows use, but does not support it in the sense of increasing its performance. This could be realized by grouping vehicles with the same origin and destination during reservation to minimize the necessary amount of merging. The transport provider's planning might also be supported by providing an overview of available slots, in addition to reacting to a specific request.

\section{Simulation Model}

To justify the advantages of trip booking, which were claimed in the previous section, simulation experiments have been performed. These experiments cover simple basic layouts to ease the validation of the results. All simulations have been performed on single-lane roads and the departure time of requests that could not be fulfilled are always shifted to a later time. Tickets are always accepted and the capacity periods have a length of $3 \mathrm{~min}$. The results of the simulations are denoted by three performance indicators, which are shown in Table II. The waiting time indicates the sum of the waiting time on the network and the waiting time at the entrance to the network. This can also be seen as the actual travel time minus the expected travel time. The 5/6th quantile of the waiting time is referred to as logistic waiting time. From a logistic point of view, this indicator is interesting because some certainty level has to be used in planning to take waiting times, resulting from stochastic fluctuations, into account [2]. In simulation experiments performed with static conditions (i.e., a constant traffic intensity), the performance indicators are determined as the average value in the stationary state. In dynamic experiments-for instance,

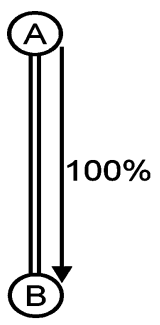

(a)

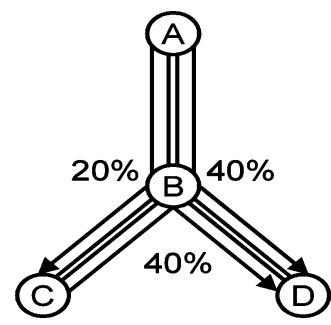

(b)
Fig. 2. Simulation configurations.

with a sine-shaped traffic intensity - the indictors are the moving average over nine measurements.

The simulation experiments compare the trip-booking and trip-metering situation with a reference situation, a situation of unorganized random vehicle arrivals. In this reference situation (i.e., unorganized arrivals), the interarrival time between two vehicles showing up at the network entrance is drawn from a negative exponential distribution. When trip booking is simulated, the desired departure times are drawn from this same distribution. The so-called anticipation time, which denotes the time between the moment when the request is made and the desired departure time, uses a uniform distribution ranging from 100 to $500 \mathrm{~min}$. The actual arrival time at the entry is also uniformly distributed, with respect to the assigned slot time, with a mean of $0 \mathrm{~min}$ and deviation of $+/-2 \mathrm{~min}$. In simulations with multiple routes, the various times for trips for each route are drawn from separate distributions. All distributions are mutually stochastically independent.

Each simulated vehicle is controlled by an individual cyclic procedure. This procedure determines a new acceleration at the start of each cycle (which has a cycle time of $0.25 \mathrm{~s}$ in the simulations). To simulate actuator lags, this acceleration is effectuated at the start of the next cycle. The speed and position of the vehicle are derived from the acceleration development. The acceleration is restricted by upper and lower bounds. The upper bounds are determined by basic vehicle characteristics, target speed, maximum jerk, (in a positive direction), and predecessor following. The lower bounds are determined by brake power and maximum jerk (in a negative direction). The vehicle following behavior is based on a constant time headway policy described in [17], which also shows that to guarantee the stability of a string of vehicles, the lags must be less than or equal to half the time headway employed. With each vehicle running its own cycle (i.e., not necessarily in phase with the others), the maximum reaction lag is $0.5 \mathrm{~s}$, consisting of $0.25-\mathrm{s}$ maximum sensing lag and 0.25 -s fixed actuator lag. As a result, a time headway of $1 \mathrm{~s}$ is sufficient. The distance between the fronts of two successive vehicles depends on the vehicle length $(15 \mathrm{~m})$, the safety distance at standstill $(1 \mathrm{~m})$, and the desired constant vehicle speed (14 $\mathrm{m} / \mathrm{s}$ ). These values result in a distance of $30 \mathrm{~m}$. The corresponding maximum road capacity is, therefore, $1680 \mathrm{veh} / \mathrm{h}$. Scaled to passenger cars, this would lead to a capacity of approximately $2500 \mathrm{veh} / \mathrm{h}$, which is not uncommon for a single-lane road with autonomous vehicles not driving in platoons [18], [19]. A merging method is applied, which results in a capacity loss in the range of $10 \%-20 \%$, which is representative of the normal situation [20].

\section{SIMULATION EXPERIMENTS}

The two configurations used for simulation are shown in Fig. 2(a) and (b). The first configuration (a) consists of a single link with all vehicles traveling the same route from A to $\mathrm{B}$. This configuration is very basic and, therefore, suited to compare the results to an analytical approach. Figs. 3 and 4 show the equilibrium results for the reference and trip-booking situations in case of a constant traffic intensity. Besides 


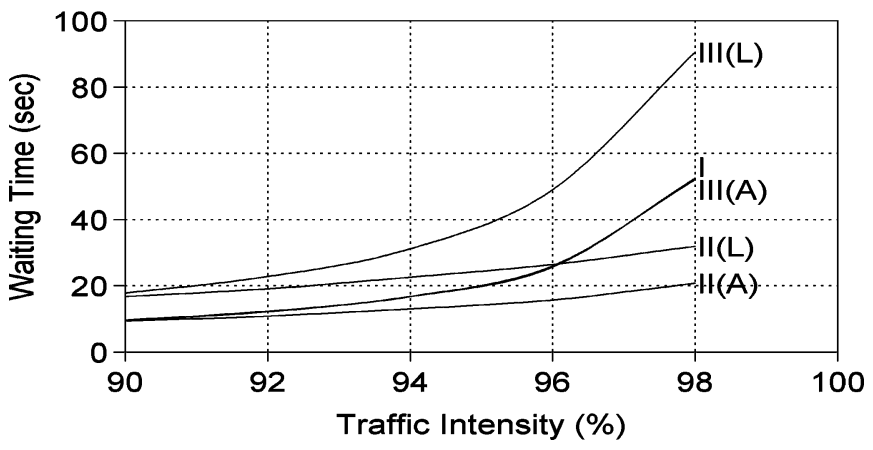

Fig. 3. Equilibrium waiting times of a static load on configuration (a). Line I refers to the result of the Pollaczek formula. Lines II(A) and II(L) correspond, respectively, to the average and logistic waiting time in the trip-booking situation. Lines III(A) and III(L) correspond, respectively, to the average and logistic waiting time in the reference situation.

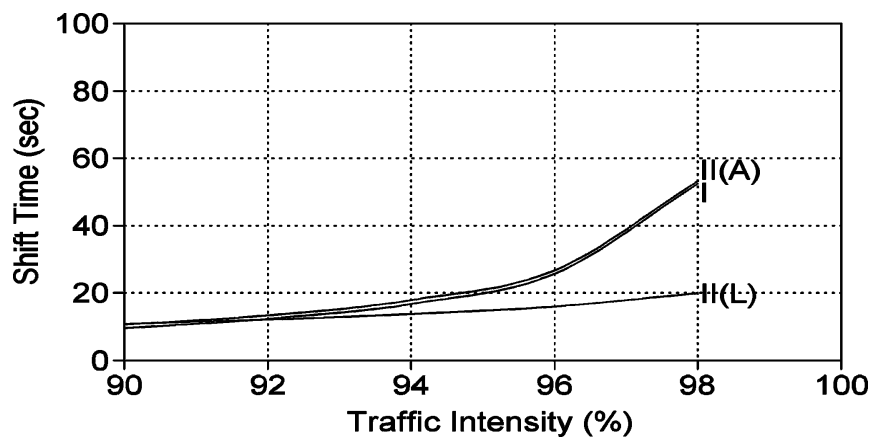

Fig. 4. Equilibrium shift times of a static load on configuration (a) Line I refers to the result of the Pollaczek formula. Lines II(A) and II(L) correspond, respectively, to the average and logistic shift time in the trip-booking situation.

the waiting times (Fig. 3), also the shift times (the difference between the desired and the allocated departure time; Fig. 4) are depicted. Analytically, this situation can be seen as a standard single-server queuing model with the road as server and the vehicles as clients. The (approximately) constant service time is determined by the vehicle following time and the service is first in, first out. For such a system, the average waiting time can be calculated by the Pollaczek-Khintchine formula [21]: $1 / 2 \cdot(\rho /(1-\rho)) \cdot(1 / \mu)$, in which $\rho$ denotes the traffic intensity (i.e., average arrival time/average service time or, in this case, traffic volume/road capacity) and $\mu$ denotes the service rate (i.e., the reciprocal of the service time or, in this case, the vehicle following time (front to front). The results of this formula are also shown in Figs. 3 and 4 . These figures show that, in the reference case, the waiting times [Fig. 3, III(A)] indeed correspond to the values predicted by Pollaczek (Fig. 3, I). In the case of trip booking, the shift times [Fig. 4, II(A)] correspond to these values, as could be expected, whereas the waiting times [Fig. 3, II(A)] result from the fact that the vehicles' actual arrival time deviates from the assigned slot time. The waiting times in the trip-booking situation appear to be significantly lower than in the reference situation. For the logistic waiting time no analytical expression is known, although it is generally larger than the average waiting time [21]. In the reference situation, this is clearly the case [Fig. 3, III(L)]. For the shift times in the trip-booking situation, this is not the case [Fig. 4, II(L)]. This is a result of the following effect. The great majority of the vehicles obtains a slot with a small departure time shift. The last vehicles that make their reservation encounter a very large departure time shift, since all the slots around their desired departure time are already filled. In the reference case, however, this would mean that the departure times of all following vehicles get shifted to a later time, resulting in the same average waiting time (compared to the

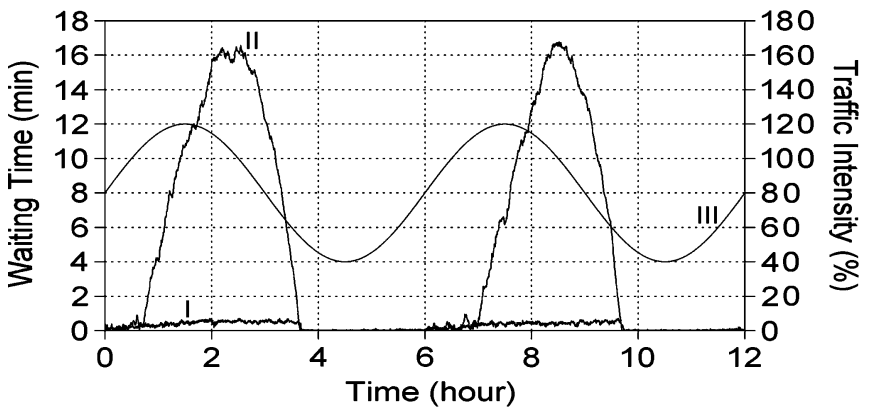

Fig. 5. Waiting times and traffic intensity in a dynamically loaded configuration (a) Line I shows the waiting time in the trip-booking situation, whereas II shows the waiting time in the reference situation (both using the left axis). Line III shows the traffic intensity (using the right axis).

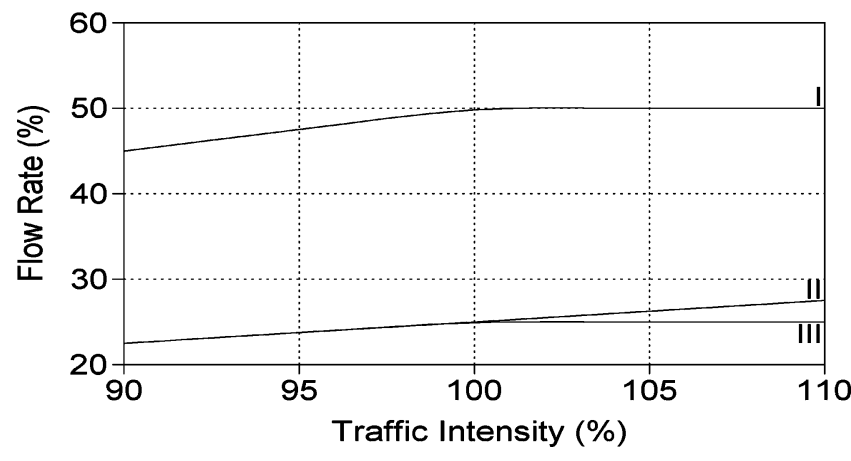

Fig. 6. Flow rate trends for a static load on configuration (b) Bottom axis refers to the traffic intensity on link BD. The left axis refers to the flow rate on the corresponding route and trend line I corresponds to routes $\mathrm{AD}$ and $\mathrm{CD}$ in all three situations. Trend line II corresponds to route AC in the trip-booking and trip-metering situations. Trend line III corresponds to route $\mathrm{AC}$ in the reference situation.

trip-booking shift time) and a higher logistic waiting time (compared to the trip-booking logistic shift time). This effect corresponds to the well-known effect that early reservations are advantageous.

Fig. 5 shows a representative example of the development of the waiting times of vehicles in the case that the traffic intensity follows a sine-shaped curve. The mean of the sine equals $80 \%$ of the road capacity and the amplitude is $50 \%$ of the mean (i.e., $40 \%$ of the road capacity). As expected, the waiting times in the nonreserved situation follow the demand with a time lag and are considerably higher than in the reserved situation. The waiting times when using trip booking are low and virtually independent of the request pattern. The flow rates in both situations are (approximately) equal.

The second configuration (b), shown in Fig. 2, represents a basic merge configuration. Vehicles travel along one of three routes: from A to $\mathrm{C}$, from $\mathrm{A}$ to $\mathrm{D}$, or from $\mathrm{C}$ to $\mathrm{D}$. The percentages indicate the relative traffic intensities on the routes. Merging at merge point $\mathrm{B}$ follows a first-come first-served principle. Simulations have been performed for the reference situation, trip-metering situation, and trip-booking situation. Only static simulations (i.e., constant traffic intensity) have been performed. The results for the flow rates are shown in Fig. 6 and the results concerning the waiting times are shown in Fig. 7. These figures depict the trends of the performance indicators around the point that the traffic volume on link BD reaches the link's capacity. From Fig. 6, it can be seen that trip metering and, therefore, also trip booking (since it includes trip metering) allows the traffic on route $\mathrm{A}$ to $\mathrm{C}$ to continue to flow, whereas in the reference case this traffic is blocked by traffic on route A to D. Fig. 7 shows that trip-metering results in small waiting times only for route $\mathrm{A}$ to $\mathrm{C}$, whereas trip booking results in small waiting times for all routes. 


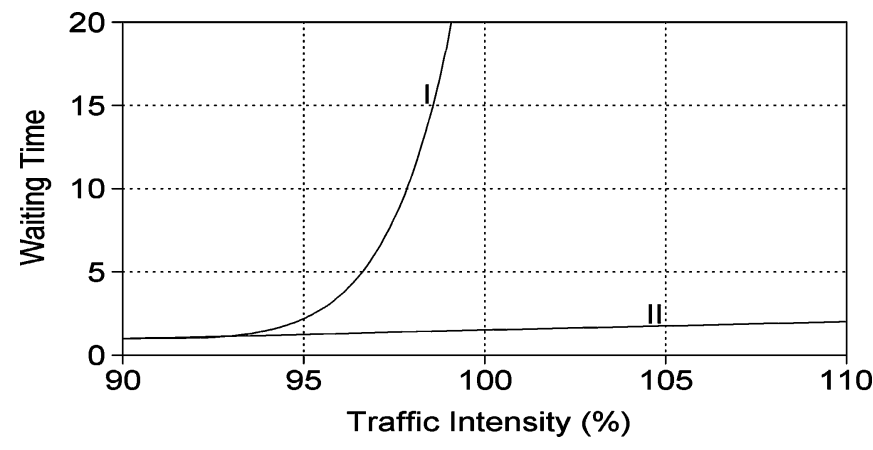

Fig. 7. Waiting time for a static load on configuration (b) Bottom axis refers to the traffic intensity on link BD. The left axis refers to the waiting time for vehicles on the corresponding route and the trend line I corresponds to all routes in the reference situation and to routes $\mathrm{AD}$ and $\mathrm{CD}$ in the trip-metering situation. Trend line II corresponds to route AC in the trip-metering situation and all three routes in the trip-booking situation.

\section{TRIP BOOKING IN A WIDER PERSPECTIVE}

Trip booking in itself is a simple concept. The application, however, can be made as complex as required by implementing case-specific allocation algorithms. Environments in which planning is of high importance, such as freight transport and public transport, seem to be a logical place to implement trip booking. This fits the current developments to create separate lanes for trucks and buses. Trip booking might, however, also be an alternative for congestion pricing [8]. It also may support the DistriRoad concept, proposed by Nederland DistributieLand [4].

The increased reliability of travel times facilitates improved transport planning. The possibility of sharing infrastructure between modalities using time restrictions has already been mentioned. Currently, a study is being performed into a concept called transport booking. This concept consists of two main parts: carrier booking to allocate transport capacity (vehicles) to transport jobs and trip booking to allocate infrastructure capacity to these vehicles. Transport booking intends to improve the performance of transport systems while preserving the autonomy of the actors: the infrastructure manager, the transport providers, and the site operators.

\section{CONCLUSION}

The simulation experiments show that trip booking produces the intended effect. It reduces waiting times, resulting in more reliable travel times. This did not require the vehicles to arrive exactly on time. Trip booking has also been shown to prevent vehicles on heavily occupied routes to block vehicles on other routes. Therefore, the flow on these routes is not restricted, resulting in a more effective use of road capacity. Both parties, the infrastructure manager as well as the transport provider, have preserved their autonomy.

This paper has presented the concept and functioning of trip booking and founded the advantage claims with simulations. These simulations have shown the basic effects of trip booking. However, more simulation experiments will be performed to quantify the results for real-world configurations. Furthermore, effects of disturbances such as capacity drops (e.g., due to accidents or bad weather) will be studied, as well as the influence of the size of the allocation periods. Simulations will also be performed concerning infrastructure shared with trains.

\section{REFERENCES}

[1] R. Bishop, Jr., "Foreword," in Automated Highway Systems, P. A. Ioannou, Ed. New York: Plenum, 1997, pp. ix-xi.
[2] T. F. Golob and A. C. Regan, "Impacts of highway congestion on freight operations: Perceptions of trucking industry managers," Transport. Res. A, vol. 35, pp. 577-599, 2001.

[3] J. J. M. Evers and R. Konings, "De slimme rail-weg hybride Betuwelijn in relatie tot het wegvervoer," Tijdschrift Vervoerswetenschap, vol. 66, no. 6, pp. 42-45, 2001. in Dutch.

[4] Nederland DistributieLand. (2002, May). Vrij baan voor personen en goederen [Online]Available: http://www.ndl.nl

[5] B. A. Pielage, "Underground freight transportation. A new development for automated freight transportation systems in the Netherlands," in Proc. IEEE Intelligent Transportation Systems, Oakland, CA, 2001, pp. 762-767.

[6] H. Akahane and M. Kuwahara, "A basic study on trip reservation systems for recreational trips on motorways," in Proc. 3rd World Congr. Intelligent Transportation Systems, 1996, pp. 1-7. ITS America.

[7] J. T. Wong, "Basic concepts for a system for advance booking for highway use," Transport Policy, vol. 4, no. 2, pp. 109-114, 1997.

[8] K. Koolstra, "Slot allocation on congested motorways: An alternative to congestion pricing," in Proc. Sem. D Held, AET Eur. Transport Conf., Cambridge, U.K., 1999, pp. 343-353.

[9] M. Zhang, T. Kim, X. Nie, W. Lin, L. Chu, and W. Recker, "Evaluation of on-ramp control algorithms," PATH, Berkeley, CA, Rep. UCB-ITSPRR-2001-36, 2001.

[10] M. Papageorgiou, C. Diakaki, V. Dinopoulou, A. Kotsialos, and Y. Wang, "Review of road traffic control strategies," Proc. IEEE, vol. 91, pp. 2043-2067, Dec. 2003.

[11] T. H. Chang and Z. Y. Li, "Optimization of mainline traffic via an adaptive coordinated ramp-metering control model with dynamic OD estimation," Transport. Res. C, vol. 12, no. 2, pp. 99-120, 2002.

[12] C. F. Daganzo, J. Laval, and J. C. Munoz, "Ten strategies for freeway congestion mitigation with advanced technologies," PATH, Berkeley, CA, Rep. UCB-ITS-PRR-2002-3, 2002.

[13] R. de Feijter, G. Lodewijks, and J. J. M. Evers, "A control framework for automated highway system operations," in 8th TRAIL Congr., 2004, to be published.

[14] D. Starkie, "Developments in transport policy: The U.S. market in airport slots," in Air Transport (Classics in Transport Analysis Series), P. Forsyth, K. Button, and P. Nijkamp, Eds. Northampton, MA: Edgar Elgar, 2002, pp. 501-505.

[15] J. T. Wong, F. S. Koppelman, and M. S. Daskin, "Flexible assignment approach to itinerary seat allocation," Transport. Res. B, vol. 27B, no. 1, pp. 33-48, 1993.

[16] A. D. May, Traffic Flow Fundamentals. Englewood Cliffs, NJ: Prentice-Hall, 1990.

[17] D. J. Bertsimas and S. S. Patterson, "The air traffic flow management problem with en route capacities," Operations Res., vol. 46, no. 3, pp. 406-422, 1998.

[18] D. Swaroop and K. R. Rajagopal, "A review of constant time headway policy for automatic vehicle following," in Proc. IEEE Intelligent Transportation Systems, Oakland, CA, 2001, pp. 65-69.

[19] R. W. Hall, A. Nowroozi, and J. Tsao, "Entrance capacity of an automated highway system," Transport. Sci., vol. 35, no. 1, pp. 19-36, 2001.

[20] D. Swaroop, J. K. Hedrick, C. C. Chien, and P. Ioannou, "A comparison of spacing and headway control laws for automatically controlled vehicles," Veh. Syst. Dynam., vol. 23, pp. 597-625, 1994.

[21] F. S. Hillier and G. J. Lieberman, Introduction to Operations Research. New York: McGraw-Hill, 2001. 